Revista Perspectivas Online: Biológicas \& Saúde Agosto de 2018, Vol.8, n 27, p. 52-62

ISSN: 2236-8868 (Online)

DOI: $10.25242 / 886882720181369$

\title{
EFEITOS DO DRY NEEDLING E DA MICROGALVANOPUNTURA SOBRE A DOR MUSCULAR NO PÓS TREINAMENTO DE FORÇA DO MÚSCULO BÍCEPS BRAQUIAL
}

\author{
Tayline Viegas Ferraz de Souzaํ, Juliana Basílio de Souza ${ }^{1}$ \& Mairkon Almeida Soares ${ }^{2}$
}

SOUZA, T.V.F.; SOUZA, J.B. \& SOARES, M.A. Efeitos do dry needling e da microgalvanopuntura sobre a dor muscular no pós-treinamento de força do músculo bíceps braquial. Perspectivas Online: Biológicas \& Saúde. v. 8 , n 27 , p.52-62, 2018.

\section{RESUMO}

Observa-se atualmente a crescente aplicabilidade do Dry Needling e Microgalvanopuntura sobre a dor musculoesquelética no pós treinamento de força muscular, porém não se encontram estudos que comprovem a eficácia das técnicas em lesões musculares. O presente estudo teve como objetivo avaliar os efeitos do Dry Needling e microgalvanopuntura sobre a dor muscular no pós-treinamento de força do músculo bíceps braquial, através de análise laboratorial de CK e Limiar de pressão a dor (PPT). A amostra foi caracterizada por 6 indivíduos do sexo masculino com média de idade de $23( \pm 3,9)$ anos, não praticantes regulares de atividade física em membro superior. Foi realizado ensaio clínico cruzado randomizado cego, no qual os indivíduos foram divididos em três grupos: Dry needling profundo com corrente microgalvânica (microgalvanopuntura), Dry needling profundo e controle, sendo realizada análise de CK e PPT. Foram utilizados banco scoot, barra com anilhas de pesos variados, medidor de pressão, striat
IBRAMED ${ }^{\circledR} \quad$ (aparelho de microgalvânica), agulha de acupuntura 0,40x0,25mm DONGBANG ${ }^{\circledR}$. Foi realizado teste de normalidade de shapirowilk com $\mathrm{p}<0,05$ e ANOVA TWO WAY - post hoc de Tukey $\alpha=$ $5 \%$..Observou-se que o protocolo de treinamento foi eficaz, CK mostra aumento 24 à $48 \mathrm{~h}$ e decréscimo $72 \mathrm{~h}$, não havendo diferença entre os grupos, sendo as técnicas de tratamento seguras, pois não variaram a expressão de $\mathrm{CK}$ em nenhum grupo de intervenção, tendo o controle o mesmo comportamento. No pós-tratamento do grupo de microgalvanopuntura, o PPT diminui, logo o indivíduo fica mais sensível ao toque na junção miotendinosa. Porém o grupo controle mostra que $24 \mathrm{~h}-48 \mathrm{~h}$ existiu diminuição do PPT, devido ao treinamento de força e não pela intervenção. Conclui-se que o método de treinamento com $80 \%$ da carga de 10 RMs é suficiente para gerar lesão em bíceps braquial, sendo as técnicas de dry needling e microgalvanopuntura seguras em lesões musculares agudas.

Palavras Chave: fisioterapia, terapia manual, bioquímica. 


\section{ABSTRACT}

It is currently observed the increasing applicability of DryNeedling and Microgalvanopuncture on musculoskeletal pain in post-muscular strength training, but no studies have been found to prove the efficacy of the techniques in muscle injuries. The aim of the present study was to evaluate the effects of Dry Needling and microgalvanopuncture on muscle pain in post-strength training of the brachial biceps muscle through laboratory analysis of CK and Pain Pressure Threshold (PPT). The sample was characterized by 6 male subjects with a mean age of 23 years ( \pm 3.9 years) who did not practice regular physical activity in the upper limb. A randomized blind crossover clinical trial was performed in which the subjects were divided into three groups: Dry needling deep with microgalvanic current (microgalvanopuncture), Deep needling deep and control, and CK and PPT analysis. Scoot bench, bar with weights of various weights, pressure gauge, IBRAMED $\AA$ striat (microgalvanic device), $0.40 \times 0.25 \mathrm{~mm}$
DONGBANG ${ }^{\circledR}$ acupuncture needle were used. A normality test of shapirowilk was performed with $\mathrm{p}<0.05$ and ANOVA TWO WAY - post hoc of Tukey $\alpha=5 \%$. It was observed that the training protocol was effective, CK showed increase 24 to $48 \mathrm{~h}$ and decrease $72 \mathrm{~h}$, there were no difference between the groups, being the treatment techniques safe, since they did not vary the expression of $\mathrm{CK}$ in any intervention group, and the control had the same behavior. In the posttreatment of the microgalvanopuncture group, the PPT decreases, soon the individual becomes more sensitive to the touch in the myotendinous junction. However, the control group showed that there was a decrease in PPT due to strength training and not intervention. It is concluded that the training method with $80 \%$ of the load of 10 RMs is enough to generate brachial biceps injuries, and dry needling and microgalvanopuncture techniques are safe in acute muscle injuries.

Keywords: physiotherapy, manual therapy, biochemistry

1 Acadêmicas do curso de Fisioterapia - ISECENSA - Rua Salvador Correa, 139, Centro, Campos dos Goytacazes, RJ, CEP:28035-310, Brasil

${ }^{2}$ Laboratório de Análise do Movimento e Prova de Função Muscular - ISECENSA- Rua Salvador Correa, 139, Centro, Campos dos Goytacazes, RJ, CEP:28035-310, Brasil

(*) e-mail: anatosplinter@gmail.com

Data de chegada: 11/06/2018 Aceito para publicação: 19/09/2018 


\section{INTRODUÇÃO}

O agulhamento seco é a utilização de agulhas finas sem a utilização de injeção, tipicamente usada para tratar a musculatura, ligamentos, tendões, fáscias, nervos, entre outros. Recentemente ele foi definido como um procedimento intramuscular, com a inserção de agulhas em nódulos nas bandas tensas musculares ou PG.

A ativação dos nociceptores periféricos acionam fibras nervosas finas do tipo III (fibras A $\delta$ ) e do tipo IV (fibras C). Devido às diferenças na velocidade de condução dos potenciais de ação das fibras A $\delta$ e $C$ é obtido a definição do termo dor rápida $(\mathrm{A} \delta)$ e dor lenta $(\mathrm{C})$, pois as fibras $\mathrm{A} \delta$ são levemente mielinizadas e as fibras $\mathrm{C}$ não são mielinizadas. Os nociceptores sinalizam quando o tecido está sendo lesionado ou corre o risco de sofrer lesão, sendo presentes em diversos tecidos, porém ausentes no encéfalo (MACHADO, 2011).

Estímulos mecânicos, químicos e térmicos excitam os receptores de dor. Essa sensação dolorosa é transmitida ao cérebro por fibras nervosas aferentes do tipo III e do tipo IV. As fibras do tipo III estão localizadas na junção do músculo com o tendão e na superfície muscular, elas identificam estímulos mecânicos. O espasmo muscular é uma resposta motora involuntária que estimula receptores de dor constantemente e causam isquemia local; portanto, o espasmo tem sido associado a uma possível causa da dor muscular tardia (TRICOLI, 2001).

A dor muscular tardia é definida como uma sensação de desconforto no músculo esquelético, ocorrendo aproximadamente 8 horas após o treino físico, aumentando sua intensidade nas primeiras 24 horas e alcançando seu pico entre 24-72 horas. Ela pode ocorrer em diferentes magnitudes e tipos de contração, tendo ênfase nas ações excêntricas. Esse tipo de contração muscular provoca microrrupturas nas fibras musculares. Essas microrrupturas geram perda da função muscular devido ao rompimento de estruturas musculares. $\mathrm{O}$ dano a essas estruturas e a deficiência no acoplamento excitação e contração são causadores da redução da força. Para obter a dimensão do dano muscular, a tensão e alongamento do músculo são definidos. Assim sendo, a ação excêntrica provoca mais lesão do que a concêntrica (ALVES, 2012).

Alguns treinos de resistência são aplicados na reabilitação, porém, as técnicas de Delorme e Oxford são as mais utilizadas. Delorme propõe um programa de exercícios de resistência progressiva (PRE) envolvendo 10 repetições máximas $(10 \mathrm{RM})$ por três séries no qual os indivíduos realizam a primeira série de 10 repetições com $50 \%$ de $10 \mathrm{RM}$, a segunda em $75 \%$ e a terceira fixada em $10 \mathrm{RM}$. Já a técnica de Oxford é regressiva, sendo realizada $10 \mathrm{RM}$ completo na primeira, reduzindo para $75 \%$ na segunda e finalizando com 50\% de 10 RM (PEREIRA et al., 2013).

Os melhores indicadores musculares são o ácido láctico, creatina quinase (CK), aspartatoaminotransferase (AST) e a lactato desidrogenase (LDH). A CK é a enzima mais específica para diagnosticar o dano muscular, pois os níveis altíssimos no plasma surgem após uma lesão muscular. A AST aumenta juntamente com a CK. No caso do $\mathrm{LDH}$, é a enzima menos específica que mantém níveis altos vários dias após a lesão muscular. Sua concentração nos eritrócitos é 150 vezes mais do que no plasma, gerando uma hemólise leve pelo seu aumento no soro. Como a LDH apresenta valores elevados por mais tempo, ela aumenta mais devagar do que a CK após a lesão muscular (PEREIRA et al., 2013).

Ações musculares como a excêntrica, concêntrica e isométrica aumentam a CK sanguínea, porém a ação excêntrica eleva mais as concentrações por gerar maior dano na fibra muscular do que nas outras ações. As maiores taxas de CK são observadas em membros superiores do que em membros inferiores, sendo explicado pelo maior uso dos músculos inferiores em atividades diárias treinando o músculo. A intensidade do exercício determina essas concentrações, sendo que a intensidade de leve a moderada não apresenta mudanças na permeabilidade da membrana. Com a intensidade alta a permeabilidade é alterada e as enzimas são liberadas para o espaço extracelular (ALVES, 2012).

Persp. online: biol. \& saúde, Campos dos Goytacazes, 27 (8), 52-62, 2018

seer.perspectivasonline.com.br 
Na literatura duas técnicas são utilizadas para tratamento de PGs e lesões musculares, o dry needling $(\mathrm{DN})$ e a microgalvanopuntura. A microgalvânica é uma corrente polarizada de baixa amperagem que acelera a produção de ATP (adenosina trifosfato) em até 500\%. Ela tem a finalidade de reparar o tecido e minimizar a dor, sendo denominada uma corrente com característica sub-umbral, onde acelera a síntese de ATP e o incremento do transporte das membranas e de aminoácidos. A estimulação microgalvânica invasiva associa seus efeitos intrínsecos da corrente com a inflamação aguda decorrente de trauma exercido pela agulha, gerando um processo de reparo tecidual. Quando a corrente é liberada há uma mudança eletroquímica entorno da agulha onde induz uma reorganização do tecido conjuntivo. Após a inserção da agulha ocorre uma leve hiperemia e edema que foram causados pelas substâncias liberadas através da lesão, vasodilatando e aumentando a permeabilidade dos vasos sanguíneos (CRUZ, 2011).

O presente estudo teve como objetivo avaliar os efeitos do Dry Needling e microgalvanopuntura sobre a dor muscular no pós-treinamento de força do músculo bíceps braquial, através de análise laboratorial de CK e Limiar de pressão a dor (PPT).

\section{METODOLOGIA}

Foi realizado um ensaio clínico cruzado randomizado cego, onde os indivíduos foram divididos em três grupos: microgalvanopuntura (DNPM), Placebo da microgalvânica, sendo o grupo de Dry needling profundo (DNP) e Controle (CTRL). A amostra do estudo foi composta por 6 indivíduos do sexo masculino, com média de idade de $23( \pm 3,9)$ anos, não praticantes regulares de exercício físico em membros superiores há no mínimo 1 mês. Foram automaticamente excluídos da coleta indivíduos que faziam uso de qualquer tipo de medicamento ansiolítico, anti-inflamatório, antidepressivo, relaxante muscular e analgésicos, com implante metálico de membros superiores, lesões musculoesqueléticas de membro superior, doenças alérgicas e infecciosas de pele, comprometimento do cegamento por lesão muscular, qualquer limitação de fazer atividade de membro superior intenso e fobia a agulha.

Todos os voluntários envolvidos na pesquisa assinaram o Termo de Consentimento Livre e Esclarecido, sendo o estudo aprovado pelo Comitê de Ética em Pesquisa do ISECENSA (CEP-ISECENSA), de acordo com os padrões para pesquisa envolvendo seres humanos, sob resolução $n^{\circ}$ 196/96, aprovada na $59^{\text {a }}$ Reunião Ordinária do Conselho Nacional de Saúde, em 10 de outubro de 1996, que instituiu os princípios para pesquisas em seres humanos, com o protocolo $\mathrm{n}^{\circ} 31102113.2 .0000 .5524$, no dia 09 de maio de 2014.

Instrumentação utilizada: banco scott, barra com anilhas de pesos variados, utilizados no teste de 10 RMs e no treino de força muscular de bíceps braquial, metrônomo que indica a momento que o voluntário deve subir e descer o braço, cronômetro que indica o tempo de descanso e de aplicação, dinamômetro de pressão, utilizado para aferição da sensibilidade, striat IBRAMED® (aparelho de microgalvânica), agulha de acupuntura $0,40 \times 0,25 \mathrm{~mm}$ DONGBANG ${ }^{\circledR}$, algodão e álcool $70 \%$ utilizado na assepsia do local da aplicação da técnica, luva descartável, tubo de sangue, seringas, torniquete, curativo, após a retirada do sangue, centrifuga (Centrífuga Basic• / Sislab Tecnologia Laboratorial Ltda - Brasil) para separar o soro utilizado na análise de $\mathrm{CK}$, pote, geladeira para guardar as amostras, pipeta para colher o material da análise, reagentes dos marcadores sanguíneos e espectrofotômetro Bioplus 200 utilizado para quantificar o resultado da análise sanguínea.

Procedimento: Foi realizado teste de 10RMs nos voluntários três dias antes de começar a coleta. O teste consistiu em quantificar o peso máximo que o voluntário conseguia levantar na rosca bíceps no banco Scott. O voluntário foi submetido a 3 series de 10 repetições com intervalo de 5 min. No período da coleta os voluntários foram orientados a não realizar atividade física de membro superior durante 4 dias.

Persp. online: biol. \& saúde, Campos dos Goytacazes, 27 (8), 52-62, 2018

seer.perspectivasonline.com.br 
Tabela 1: Ordem cronológica da coleta de dados e intervenção

\begin{tabular}{|c|c|c|c|}
\hline PRIMEIRO DIA & SEGUNDO DIA & TERCEIRO DIA & QUARTO DIA \\
\hline $\begin{array}{l}\text { COLETA DE } \\
\text { SANGUE }\end{array}$ & COLETA DE SANGUE & $\begin{array}{l}\text { COLETA DE } \\
\text { SANGUE }\end{array}$ & COLETA DE SANGUE \\
\hline PPT & PPT & PPT & PPT \\
\hline TREINAMENTO & TRATAMENO & & \\
\hline PPT & PPT & & \\
\hline
\end{tabular}

PPT: LImiar de dor a pressão

Coleta de sangue (fig.: 1): para análise de CK, o sangue foi coletado $(+\backslash-5 \mathrm{ml}$ ) na fossa cubital dos indivíduos, que estavam sentados no momento da coleta. Foram utilizados materiais descartáveis (agulhas e seringas). Após a coleta, o sangue foi depositado em recipientes fechados de vidro, sendo imediatamente levado para o Laquibio do Instituto Superior de Ensino do Censa (ISECENSA-RJ), onde foi centrifugado (Centrífuga Basic / Sislab Tecnologia Laboratorial Ltda - Brasil) para separar o soro. Para se determinar os níveis de CK, foi utilizado o kit de reagente CK - NAC (Labtest Diagnóstica SA - Brasil), e um analisador automático espectrofotômetro Bioplus 200 utilizado para quantificar o resultado da análise sanguínea onde foi utilizada uma média triplicata dos resultados.

O PPT foi realizado com o voluntário em DD, na junção miotendinosa do músculo bíceps braquial, onde o terapeuta fez uma força em direção ao músculo, com um dispositivo adaptado em libras/força, quando o voluntario relatava dor, era mensurado o valor da sensibilidade, feito uma média triplicata dos valores. A conversão dos valores foi feita de 1libra/força para $0,14 \mathrm{KgF}$.

Treinamento (fig.1): treinamento de força que consistiu de $80 \%$ da carga máxima do protocolo de 10 RMs para rosca bíceps em banco Scott com 5 series até o limite máximo do voluntário com intervalo de 1 min entre as series, utilizando o metrônomo para indicar o momento de subida e descida do braço durante o exercício, com intervalo de descanso de 1 minutos pelo cronometro.

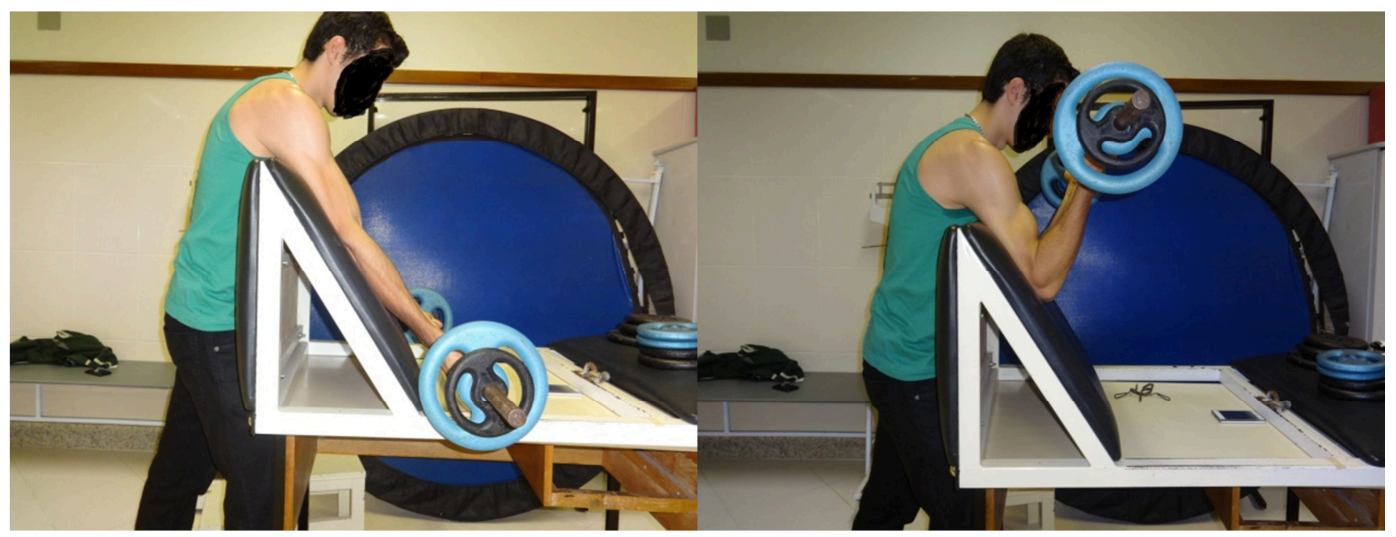

Figura 1- Protocolo de treinamento de força para bíceps braquial 
Os voluntários foram randomizados através de sorteio, nos grupos DNPM (Dry needling com microcorrente), PLACEBO (Dry needling profundo) e CTRL (Controle). A técnica da aplicação da agulha no tratamento: agulha de $4 \mathrm{~cm}$ com penetração de $1,5 \mathrm{~cm}$, insere a agulha distal $45^{\circ}$ graus sentido cranial e a agulha proximal $45^{\circ}$ graus sentido caudal, realiza manobra de agulha até gerar contração do músculo.

DNPM (fig 2): aplicação da agulha na junção miotendinosa do músculo bísceps braquial onde o paciente relatava sentir dor e a outra proximal paralelo a primeira. No posicionamento dos eletrodos, o catodo na parte distal e o anodo na parte proximal do músculo com intensidade de $160 \mu \mathrm{A}$ por 20 minutos em cada membro.

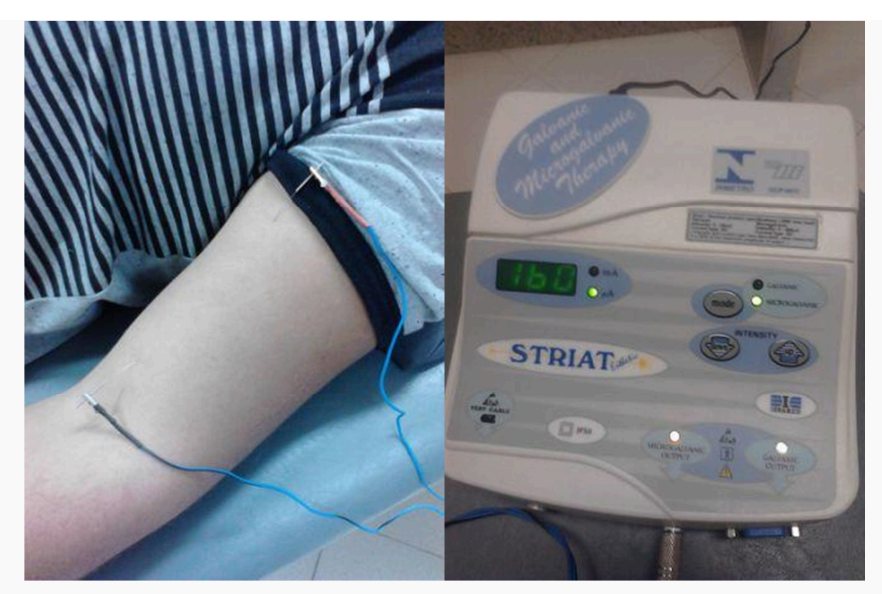

Figura 2 - Tratamento de Dry needling com microcorrente

PLACEBO (fig 3) (Dry needling), aplicação da agulha na junção miotendinosa, onde o paciente relata sentir dor e outra proximal paralelo a primeira. Foram posicionados os eletrodos com o equipamento em dose de $0 \mu \mathrm{A}$ no tempo de 20 min em cada membro.

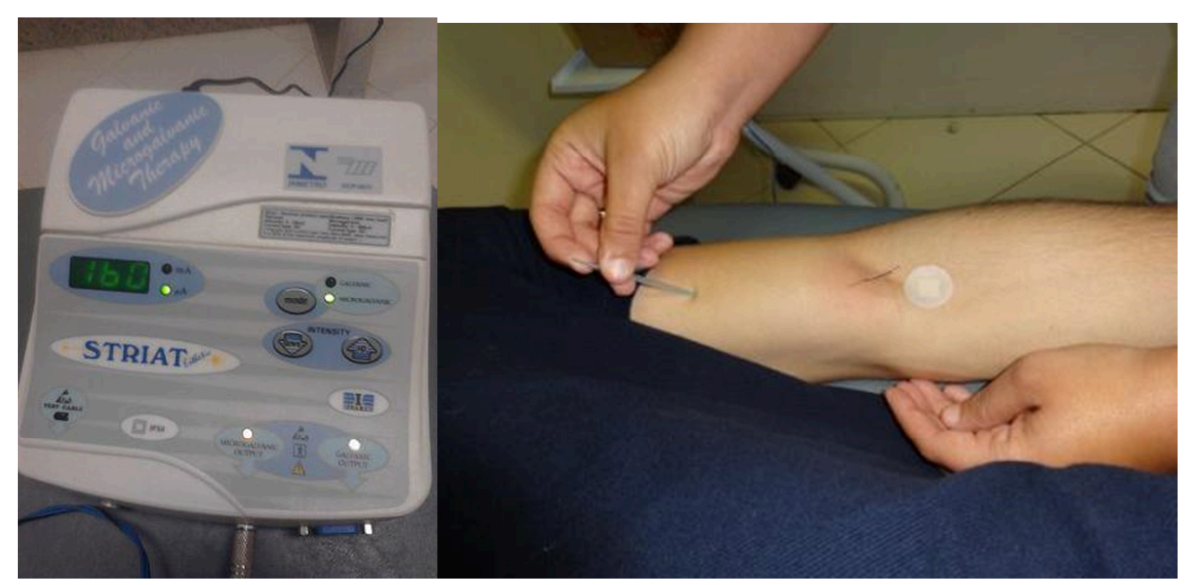

Figura 3 - PLACEBO (Dry needling profundo) sem intensidade no equipamento

CTRL (controle): Os indivíduos foram submetidos ao mesmo método de treinamento, sendo realizada apenas as coletas sanguíneas e PPT. Os voluntários tiveram um intervalo de 1 mês para serem submetidos aos grupos descritos. 


\section{RESULTADOS}

A figura 4 mostra Delta CK (sendo este a média da diferença do pico pelo basal, sendo $p>0,05$ no teste ANOVA TWO WAY - post hoc de Tukey.) nos grupos de intervenção e controle, após treinamento de força com $80 \%$ do protocolo de 10 RMs para o músculo bíceps braquial. Obteve no grupo de dry needling profundo valor de delta CK de $220 \mathrm{U} \backslash \mathrm{L}( \pm 250)$, no grupo de microgalvanopuntura valor $380 \mathrm{U} \backslash \mathrm{L}( \pm 240) \mathrm{e}$ grupo controle valor $400 \mathrm{U} \backslash \mathrm{L}( \pm 220)$, onde os grupos em relação ao controle não observam-se variação.

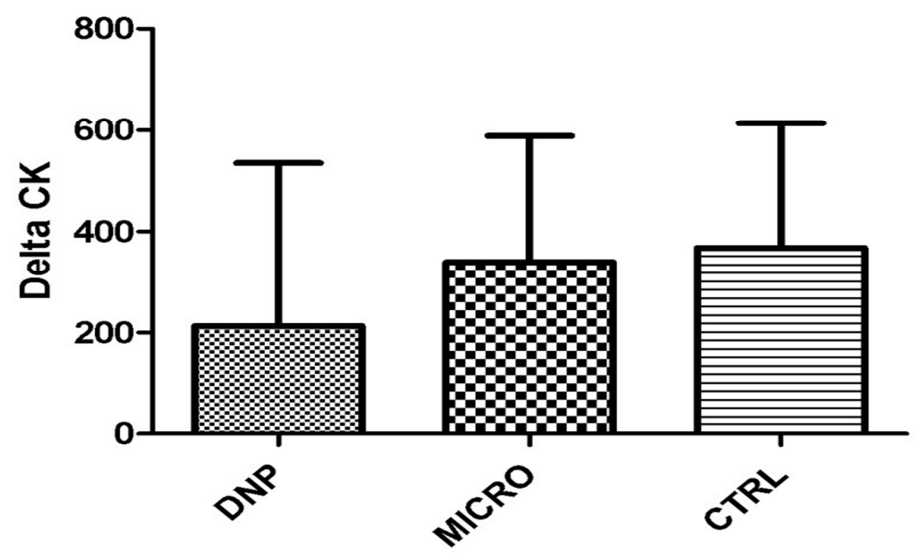

Figura 4 - Comportamento dos níveis de Delta CK em percentual nos grupos de dry needling profundo (DNP), microgalvanopuntura (micro) e controle (ctrl)

Na figura 5, mostra CK (\%), onde o momento basal analisou CK sem atividade de força, $24 \mathrm{hs}$ sendo pós-treinamento, $48 \mathrm{hs}$ representando $24 \mathrm{hs}$ pós-tratamento e $72 \mathrm{hs}$ representando $48 \mathrm{hs}$ pós-tratamento. observa-se aumento da CK $(\%)$ no grupo de dry needling profundo no momento basal $-100 \%$ em relação à $24 \mathrm{hs}-300 \%( \pm 100)$, tendo no grupo de microgalvanopuntura um aumento de CK no momento basal $100 \%$ e $24 \mathrm{hs}-320 \%$ ( \pm 120$)$ e no grupo controle um aumento de basal $-100 \%$ e $24 \mathrm{hs} 680 \%-( \pm 370)$.

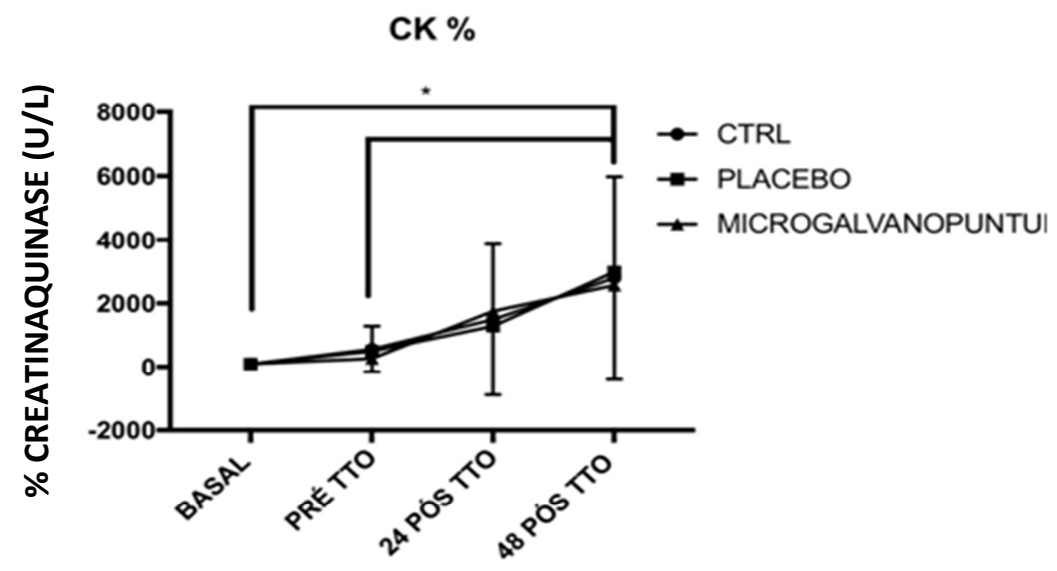

Figura 5 - Comportamento dos níveis de CK normalizados nos Grupos estudados; placebo (DNP) - dry needing profundo, DNPM - microgalvanopuntura e CTRL - Controle. (*) Indica diferença entre basal - pré tto e 48 hs pós tto nos três grupos $(* p<0,05)$ no teste ANOVA TWO WAY - post hoc de Tukey. 
$\mathrm{Na}$ figura 6, apresentam-se os resultados médios do PPT, no grupo Dry needling profundo. Observou-se diferença de $24 \mathrm{hs}$ pós tratamento em relação ao pré tratamento e basal, onde diminuiu o limiar de sensibilidade observando respectivamente basal $-16 \mathrm{kgf}( \pm 7), 24 \mathrm{~h}$ pré tratamento $-14 \mathrm{kgf}( \pm 7)$ e $24 \mathrm{~h}$ pós tratamento $-12 \mathrm{kgf}( \pm 2)$.

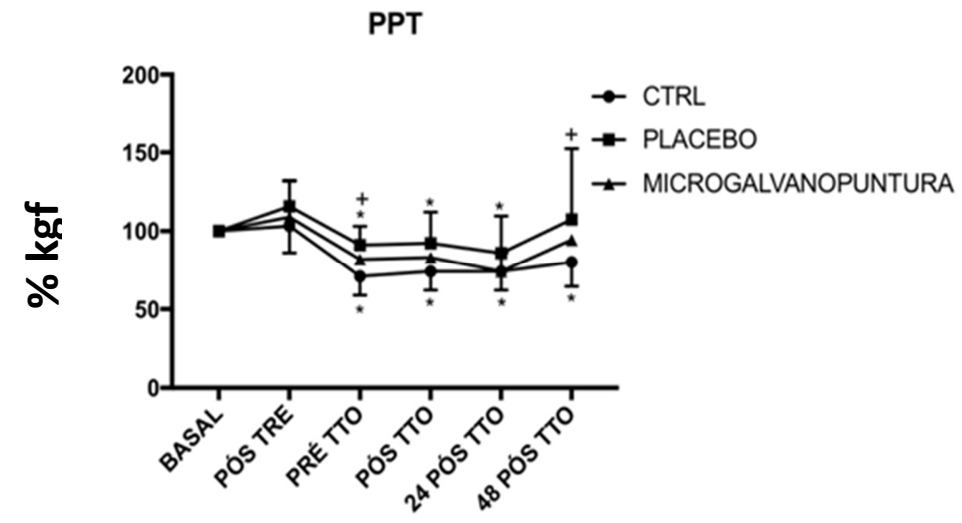

Figura 6- Valores médios normalizados de pressão (KgF) no grupo placebo (DNP), microgalvanopuntura e controle sendo ANOVA TWO WAY - post hoc de Tukey $(* \mathrm{p}<0,05)$ diferença intragrupo para os momentos em ralação ao basal e $(+p<0,05)$ diferença intergrupo durante os momentos especificados.

\section{DISCUSSÃO}

O protocolo de treinamento de 10 RMs de $80 \%$ da carga foi suficiente para gerar lesão em bíceps braquial, com aumento expressivo de CK\% não havendo diferença entre os grupos, sendo demonstrado neste trabalho na figura 5. No estudo com indivíduos sem experiência em treino de força verificou-se um aumento expressivo da concentração de CK 24 horas após o primeiro teste de força, pois a enzima CK extravasa para o meio extracelular após dano em fibras musculares por não ter a capacidade de atravessar a barreira da membrana sarcoplasmática. Esse aumento na concentração sérica se torna um marcador indireto de dano muscular (FOSCHINI et al, 2007).

Em outro estudo foi realizado dois protocolos de treinamento de força, sendo que ambos os grupos realizariam 10 séries de 10 repetições $(10 \mathrm{RM})$ a $65 \%$ de $1 \mathrm{RM}$. Um grupo realizou o treinamento com intervalo entre as séries de 1 minuto e o outro com intervalo de 3 minutos. No grupo de 1 minuto de intervalo, a concentração de CK aumentou 24 horas após o término do treino, já no grupo de 3 minutos não houve diferença significativa, sugerindo que o tempo de intervalo entre as séries interfere no dano muscular. Tendo neste trabalho séries utilizadas com intervalo de 1 min (PEREIRA, 2013).

Um estudo em que sete homens com idade na média de 22 anos, foram submetidos à realização da técnica de Delorme e Oxford para flexores do cotovelo, com 8-10 repetições em 3-4 séries de 75\%-85\% de 1 RM com 1-2 minutos de descanso entre as séries. Não houve diferença significativa entre os dois grupos, mostrando que há lesão igual em ambos (PEREIRA, 2013).

Estudos mostraram que a prática do exercício excêntrico inabitual gera lesão muscular, tendo sintomas como redução da amplitude de movimento (ADM), perda de força, DMT, inchaço do membro e 
concentração sérica de CK. Os principais fatores que influenciam a magnitude da lesão é o número de contrações excêntricas, grau de estiramento e tensão produzida. Pesquisas recentes relatam que a velocidade de contração em uma sessão do treino excêntrico dos músculos flexores de cotovelo influencia a magnitude e recuperação de alguns marcadores indiretos de lesão. Ao realizarem de forma rápida, o rompimento da linha $\mathrm{Z}$ mostrou maior lesão que de forma lenta (ELLWANGER et al., 2007).

Segundo dados da literatura, no tecido saudável há fluxo de correntes elétricas pelo organismo. Quando esse fluxo é alterado no tecido é lesionado a corrente elétrica estimula a reparação tecidual. A terapia com microcorrente tem por objetivo normalizar o fluxo de correntes, acelerando o reparo e minimizando a dor (MIYASHITA, 2012).

Na microgalvanopuntura se utiliza agulhas associadas aos efeitos do pólo negativo da corrente galvânica. $\mathrm{O}$ estímulo físico da agulha juntamente com a alcalose originada no pólo negativo da corrente contínua, promove a inflamação aguda. A inflamação gera vasodilatação aumentando o fluxo sanguíneo. $\mathrm{O}$ sangue, linfa e a água contêm íons de sódio, cálcio e cloretos que conduzem a corrente elétrica que secundariamente ocorre à eletrólise, desencadeando uma quebra de substâncias condutoras em seus componentes, processo este que ocorre em tecidos como músculos, ossos e nervos. $\mathrm{O}$ aumento na permeabilidade vai gerar edema, que é sinal característico do processo inflamatório decorrente do uso da microcorrente galvância com agulhas (REBONATO et al., 2012).

Muitos neurotransmissores têm sua ação particularmente alterada pela estimulação elétrica. $\mathrm{O}$ agulhamento atua no portão da dor, havendo evidências de que estimula a produção de endorfinas, serotonina e acetilcolina no sistema nervoso central, aumentando a analgesia. Em discordância com o presente estudo, Furlan (2011) verificou no grupo onde foi utilizado a microcorrente, 24 horas póstratamento há uma piora da sensibilidade local, devido às células estarem mais excitadas. Este estudo utilizou a microcorrente com 160 microamperes, corroborando com Silva et al (2014) onde relatam que correntes de 100 microamperes a 500 microamperes aumentam a síntese proteica de $30 \%$ a $40 \%$.

Dommerholt et al (2006) relata que a dissolução de um ponto gatilho sensibilizado realizada pelo agulhamento seco profundo reproduz queixa de dor pelos pacientes, sendo relativamente fácil desencadear um padrão de dor ao ser comparado com técnicas manuais. Pelo fato do agulhamento em pontos gatilho ser invasivo é difícil implantar estudos randomizados controlados e com placebo. Alguns estudos clínicos relatam que o agulhamento seco em pontos gatilho imediato causa analgesia em quase $87 \%$ dos locais da agulha, constatou que o agulhamento seco profundo é mais eficaz em curto prazo na redução da dor.

Chonan (2008) revela que houve uma diminuição geral das medidas do algômetro pós-agulhamento, o que indica aumento da sensibilidade no local tratado por agulha até 24 pós tratamento, apresentando diferença intergrupo em $48 \mathrm{hs}$ pós tratamento, mostrando melhora do grupo placebo (DNP). A quantitificação de CK é utilizada como um reflexo da quantidade de dano tecidual, que começou a aumentar duas horas pós agulhamento e atingiu um máximo em 12 horas. Porém o presente estudo revela que o no grupo controle houve piora da sensibilidade local, sendo esta atribuída ao treinamento e não a utilização da agulha ou da microcorrente, pois no intervalo entre $24 \mathrm{~h}-48 \mathrm{~h}$ ocorreu a diminuição da sensibilidade e nos grupos tratados, não houve variação de $\mathrm{CK}$, observando-se uma maior segurança para aplicação de dry needling e da microgalvanopuntura em lesões agudas.

No placebo (DNP) a profundidade da agulha sobre o tecido é de 10 a $15 \mathrm{~mm}$. Ao aplicar a agulha sobre a pele no ponto gatilho, estimula-se a fibra nervosa $\mathrm{A} \delta$, o que leva ao bloqueio da transmissão da dor, que pode ser imediato ou durante várias horas. Esse estudo revela isso, ao aplicar a agulha o voluntário deixa de sentir dor. Através da análise de CK comprovou-se isso, pois não observou variação entre os grupos, logo a dor que o voluntário sentiu foi do exercício e não do agulhamento ou da corrente pois o grupo controle, onde não aconteceu nenhuma intervenção, obteve-se o mesmo resultado de CK dos grupos onde ocorreram intervenções.

Persp. online: biol. \& saúde, Campos dos Goytacazes, 27 (8), 52-62, 2018

seer.perspectivasonline.com.br 
Logo pode-se sugerir que a técnica é segura e eficaz no alivio da dor na fase aguda. Em seu estudo observa-se o efeito do agulhamento profundo em dor no ombro hemiparético em sobreviventes de AVC. O resultado foi excelente no alivio da dor, onde todos os pacientes tiveram inclusive melhora no sono, conforto em suas cadeiras de rodas e durante a fisioterapia. Nesse estudo nota-se uma limitação no número de voluntários, necessitando de mais estudos para observar o comportamento das amostras.

\section{CONCLUSÃO}

Conclui-se que a utilização da metodologia proposta permitiu verificar que a aplicabilidade do método de treinamento com $80 \%$ da carga de 10 RMs foi suficiente para gerar lesão em bíceps braquial, mostrando aumento da CK 24 hs pós-treinamento, não havendo diferença entre os grupos. Já no PPT houve diminuição do limiar de sensibilidade à dor $24 \mathrm{hs}$ pós-tratamento em todos os grupos, mostrando que a aumento da dor na junção miotendinosa foi devido ao treinamento, não ocorrendo contribuição da metodolia proposta. Sendo assim, este estudo mostra que as técnicas de microgalvanopuntura e dry needling profundo não aumentam lesões musculares agudas. Propõe-se que novos estudos devem ser realizados, com um número de amostra maior para melhor observação dos resultados.

\section{REFERÊNCIAS}

ALVES, A. Comportamento da enzima creatina quinase sanguínea em jogadores de futebol de elite durante o campeonato brasileiro; Belo Horizonte, 2012.

ALVES, R. Ponto-gatilho miofacial: histórico e métodos de identificação; Porto Alegre, 2011.

BALDRY P., Superficial versus deep dry needling, Acupunture in medicine; 2002;20(2-3):78-81.

CHONAN, D. The effect of cryotherapy on post dry needling soreness, 2008.

CRUZ, J. Indicadores bioquímicos da função muscular; Rio Grande do Sul, 2011.

DILOREZO, Hemiparetic Shoulder Pain Syndrome Treated with Deep Dry Needling During Early Rehabilitation: A Prospective, Open-Label, Randomized Investigation, Journal of Musculoskeletal Pain, Vol. 12(2) 2004.

DUNNING, J. et al. Dry needling: a literature review practice guidelines, Physical Therapy Reviews; 2014, VOL. 000, NO. 000.

ELLWANGER, R. et al. Efeito da utilização de diferentes velocidades do treino de força em marcadores indiretos de lesão muscular, Rev. bras. Educ. Fís. Esp.; São Paulo, v.21, n.4, p.259-70, out./dez. 2007.

FOSCHINI, D. et al. Relação entre exercício físico, dano muscular de início tardio, Rev. Bras. Cineantropom. Desempenho Hum. 2007; 9(1):101-106.

GALDINO, A. et al. Análise comparativa do efeito da corrente microgalvânica: estudo de caso no tratamento de estrias atróficas;Revista Eletrônica "Saúde CESUC" - Centro de Ensino Superior de Catalão, Ano I, No 01 $-2010$.

GURJÃO, A. et al. Variação da força muscular em testes repetitivos de 1 RM em crianças pré-puberes; RevBrasMed Esporte _ Vol. 11, No 6 - Nov/Dez, 2005.

KALICHMAN L. et. al., Dry needling in the management of musculoskeletal pain, clinical review, JABFM September-October 2010 Vol. 23 No. 5.

LIBARDI, C. et al. Comparação de testes de 1 RM e 10 RM em homens jovens treinados; SAÚDE VER; Piracicaba, 9 (22): 31-37, 2007.

MACHADO, A. Análise da relação entre parâmetros da onda-M e a dor; Uberlândia, 2011.

MANESCO, C. Fisiologia da dor muscular tardia; Piracicaba, 2010. 
MARTIGNAGO, C. et al. Microcorrente galvânica como recurso fisioterapêutico para o tratamento de estrias albas, Anais do XVIII EAIC. 2009.

MCMAKIN, C. Microcurrent therapy: a novel treatment method for chronic low back myofascial pain, Journal of Bodywork and Movement Therapies, 2004, 8, 143-153.

MUTUBERRIA, M. Utilização da microcorrente e ultra-som terapêutico pulsado na cicatrização de úlcera diabética; Novo Hamburgo, 2006.

PEREIRA, W. et al. Influência da diatermia por micro-ondas na força dos músculos flexores do cotovelo e na atividade eletromiográfica do músculo bíceps braquial. Fisioter. Mov. 2008 jul/set;21(3):27-35.

SILVA, R. et al. Efeitos da microcorrente sobre o tecido conjuntivo de ratos Wistar. Fisioterapia Brasil, volume 15, número 4, julho/agosto de 2014.

SONNEWEND, D. et al. O efeito da radiação infravermelho longo e microcorrentes sobre o processo de reparação de feridas em ratos. VIII Encontro Latino Americano de Iniciação Cientifica e IV Encontro Latino Americano de Pós-Graduação - Universidade do Vale do Paraíba, 2004.

TEIXEIRA, M. et al. Fisiopatologia da dor músculo-esquelética.Rev. Med. São Paulo, 80 ed. esp. pt.1:63-77, 2001.

TRICOLI, V. Mecanismos envolvidos na etiologia da dor muscular tardia, Rev. Bras. Ciên. e Mov. Brasília, v. 9 n. 2 p. $39-44$ abril 2001.

ZIAEIFAR, M. et al. The effect of dry needling on pain, pressure pain threshold and disability in patients with a myofascial trigger point in the upper trapezius muscle. Journal of Bodywork \& Movement Therapies, $2014,18,298-305$. 